\title{
Recurrent acute pulmonary toxicity and respiratory failure associated with fludarabine monophosphate
}

\author{
Corey Cutler MD, Yaseen Samman MD, Richard Fraser MD, Paul Corris MD, Arnold Zidulka MD \\ Department of Medicine and Department of Pathology, Royal Victoria Hospital, \\ Montreal, Quebec
}

C Cutler, Y Samman, R Fraser, P Corris, A Zidulka. Recurrent acute pulmonary toxicity and respiratory failure associated with fludarabine monophosphate. Can Respir J 1997;4(6):331-333.

A case of recurrent acute fludarabine pulmonary toxicity in a 67-year-old man with non-Hodgkin's lymphoma is presented. The patient responded well to high dose steroid therapy. The literature with respect to this rare entity is reviewed.

Key Words: Fludarabine monophophate, Non-Hodgkin's lymphoma, Pulmonary toxicity
Toxicité pulmonaire aiguë et insuffisance respiratoire associées au monophosphate de fludarabine

RÉSUMÉ : Un cas de toxicité pulmonaire aiguë et récurrente attribuable à la fludarabine chez un homme de 67 ans atteint d'un lymphome non hodgkinien est présenté. Le patient a bien répondu à un traitement par stéroïdes à hautes doses. La littérature concernant cette rare entité est passée en revue.
$\mathrm{F}$ udarabine monophosphate (9-beta-D-arabinofuranosyl2-fluoroadenine-5'-monophosphate) is a synthetic purine analogue, related to both the antileukemic pyrimidine analogue, cytarabine, and to the antiviral agent, vidarabine. Unlike vidarabine, fludarabine is resistant to deamination in vivo by the enzyme adenosine deaminase, making it an active antineoplastic agent. Fludarabine is effective in the treatment of lymphoid malignancies including chronic lymphocytic leukemia and non-Hodgkin's lymphoma $(1,2)$.

The most common adverse effect of fludarabine is myelosuppression with neutropenia. Less common adverse effects include neurotoxicity, nausea, vomiting, diarrhea, somno- lence and transient elevations in hepatic enzymes or creatinine. Pulmonary toxicity has been previously incidentally reported in the literature (1), in abstract format $(3,4)$ and as case reports (5-7). We report a life-threatening case of recurrent fludarabine-associated pulmonary toxicity requiring mechanical ventilation occurring 24 days after receiving fludarabine monotherapy for relapsed non-Hodgkin's lymphoma.

\section{CASE PRESENTATION}

In 1987, a 67-year-old man with a history of asbestos exposure and a major affective disorder presented with painless cervical and axillary lymphadenopathy. This was diagnosed 


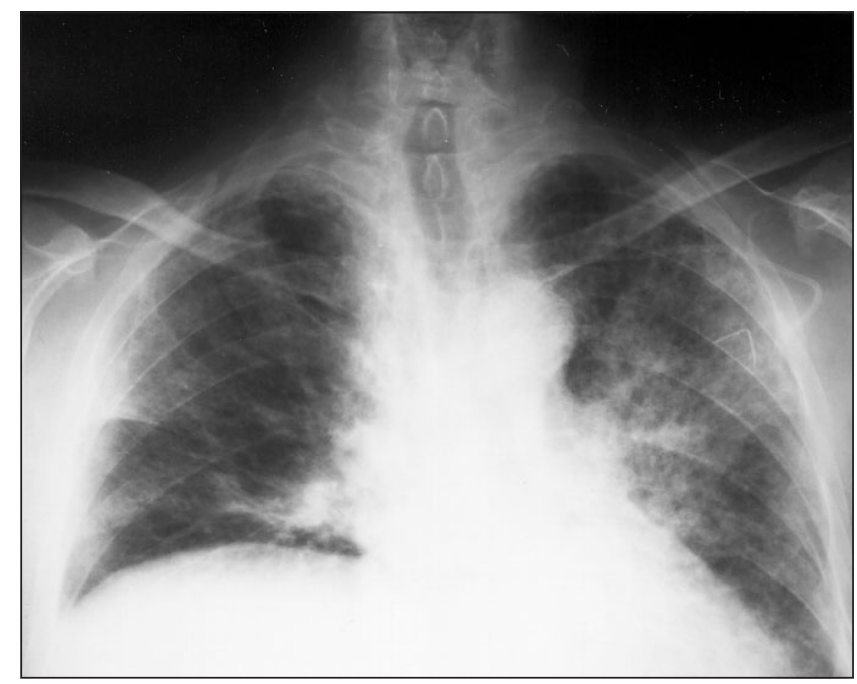

Figure 1) Initial chest radiograph showing bilateral interstitial pulmonary disease, more prominent on the left and an elevated right hemidiaphragm

as non-Hodgkin's lymphoma, small noncleaved cell type. He had a 40 pack-year smoking history and no known allergies, and was taking only lithium and fluoxetine for his affective disorder. The patient received combination chemotherapy from 1987 to 1989 and from 1992 to 1994 with the following agents according to standard protocols: etoposide, doxorubicin, cyclophosphamide, vincristine, bleomycin and prednisone. Intrathecal methotrexate and cytosine arabinoside were administered in 1989 . He also received radiation therapy to the right cervical region in 1994, the therapy causing a phrenic nerve injury. Due to relapsed mediastinal and para-aortic lymphoma in March 1995, the patient received fludarabine $\left(25 \mathrm{mg} / \mathrm{m}^{2}\right.$ intravenously for five consecutive days) and was discharged from hospital without complications. He was readmitted April 21, 1995, complaining of increasing shortness of breath and cough over the previous two weeks. A chest radiograph and computed tomography of the chest at that time were unchanged compared with previous studies. Supplemental oxygen and methylprednisolone - 100 $\mathrm{mg}$ daily for three days, followed by prednisone at an initial dose of $60 \mathrm{mg}$ per day and tapering over two weeks - were given for presumed increased airflow obstruction, and the patient's dyspnea improved. Before being released from hospital, he received a second course of fludarabine, $25 \mathrm{mg} / \mathrm{m}^{2}$ (May 1 to May 5, 1995).

The patient was readmitted May 29, 1995 with a three-day history of fever, nonproductive cough and shortness of breath. On examination at this time, the patient was pyrexic $\left(38.9^{\circ} \mathrm{C}\right)$, tachypneic and had an oxygen saturation of $81 \%$ and a $\mathrm{PaO}_{2}$ of $42 \mathrm{mmHg}$ on a rebreathing mask with a fractional concentration of inspired oxygen of 1.0. He had diffuse coarse crackles to the midlung fields. There was no clinical evidence of heart failure. All routine hematological and biochemical parameters were within normal limits. Specifically, the patient was neither neutropenic nor lymphopenic during his admission. A chest radiograph showed bilateral interstitial pulmonary disease, more prominent on

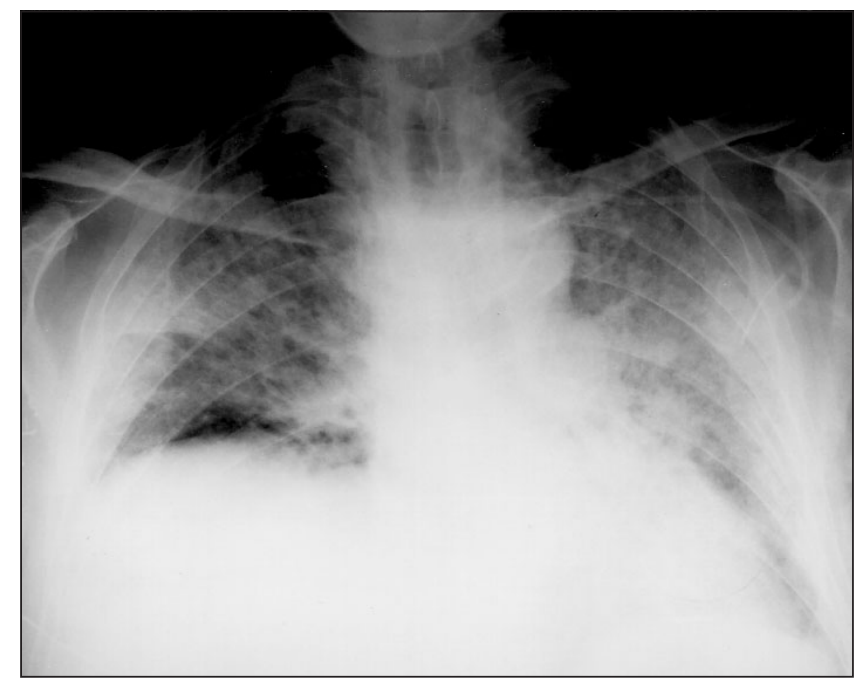

Figure 2) Chest radiograph performed one day after admission showing progression to diffuse pulmonary air-space disease and the elevated right hemidiaphragm

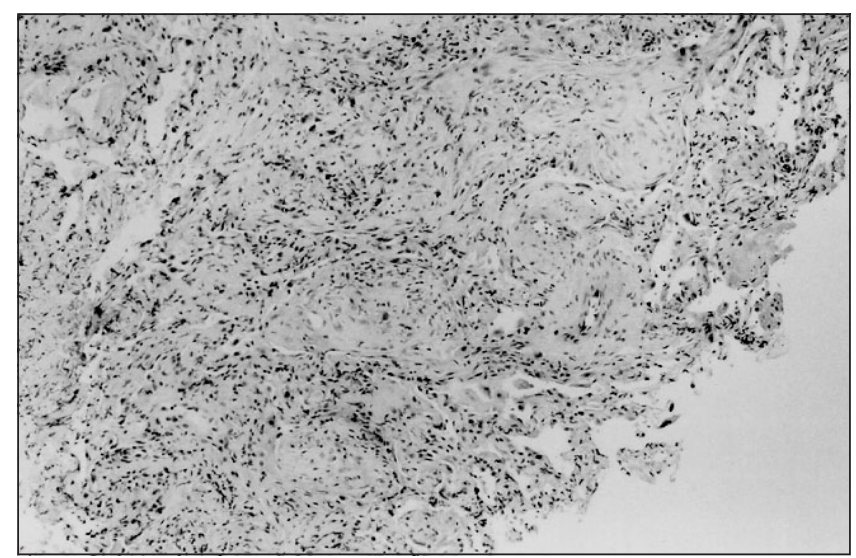

Figure 3) Biopsy specimen showing active fibrosis of airspaces and interstitium consistent with the organizing phase of diffuse alveolar damage

the left, and a chronically elevated right hemidiaphragm (Figure 1). He received broad spectrum antibiotics, exhibiting no response. By day 2, there was radiographic evidence of diffuse airspace disease (Figure 2). The patient was intubated and mechanically ventilated the next day. Bronchoscopy with bronchoalveolar lavage and transbronchial biopsy was performed. The biopsy specimen revealed an organizing proteinaceous exudate within alveolar airspaces, reactive type 2 pneumocytes and a moderate degree of interstitial fibrosis, consistent with the organizing phase of diffuse alveolar damage. There was no evidence of malignancy, and no asbestos bodies, intracytoplasmic inclusions or microorganisms, including Pneumocystis carinii, were seen (Figure 3). All cultures of bronchial washings were negative for bacteria, fungi and mycobacteria.

High dose corticosteroid therapy (methylprednisolone $40 \mathrm{mg}$ intravenously tid) was administered for presumed fludarabine toxicity. He was taken off the ventilator three days later with improvement of the radiographic picture. He was discharged June 16, 1995 on $60 \mathrm{mg}$ of prednisone per 
day to be tapered over several months. His chest radiograph slowly returned to baseline after several months, and no further fludarabine was given.

\section{DISCUSSION}

This case report and three others reported in the literature (5-7) support the association of fludarabine therapy and acute pulmonary toxicity. The presenting symptoms of chemotherapy-induced pulmonary toxicity caused by different offending agents are fairly constant and include fever, nonproductive cough and dyspnea. Fever is a prominent finding; it may predate or antedate the pulmonary manifestations, but is almost universally present and is rarely associated with chills or rigors. The level of dyspnea and hypoxemia depend highly on the level of induced toxicity (8).

The clinical signs and symptoms of this patient were similar to those described in other reports of fludarabine toxicity (5-7). Radiographic findings in fludarabine toxicity consist of interstitial pulmonary disease occasionally progressing to airspace disease, as occurred in this patient. Pleural effusions have been noted in two patients (1). Several nonspecific pathological changes have been described, including hypersensitivity pneumonitis (eosinophilic pneumonia), interstitial pneumonitis and diffuse alveolar damage, as this patient had. In all other reported cases, the patients had been previously exposed to chlorambucil, an agent with known pulmonary toxicity (8). The patient reported here, however, had no exposure to this agent, but had received bleomycin, a known pneumotoxin (8).
In this case, possible fludarabine toxicity initially developed three weeks after the termination of a first course of therapy and was inadvertently treated with corticosteroids for presumed increased airflow limitation. After a second course of fludarabine, a much more severe form of pulmonary toxicity appeared. This began with interstitial disease and progressed to airspace disease for which the patient required mechanical ventilation (reported only once previously [7]). Rapid resolution of disease and clearance of radiographic findings after steroid therapy in this case provide support for the use of high dose corticosteroids in cases of fludarabine toxicity.

\section{REFERENCES}

1. Chun HG, Leyland-Jones B, Cheson BD. Fludarabine phosphate: A synthetic purine antimetabolite with significant activity against lymphoid malignancies. J Clin Oncol 1991;9:175-88.

2. Cheson BD. New chemotherapeutic agents for non-Hodgkin's lymphoma. Hematol Oncol Clin North Am 1991;5:1027-53.

3. Grever MR, Coltman CA, Files JC, et al. Fludarabine monophosphate in chronic lymphocytic leukemia. Blood 1986;68:223A. (Abst 771)

4. Mittleman A, Lichtman S, Budman D, et al. Therapy of chronic lymphocytic leukemia (CLL) with fludarabine monophosphate (FAMP). Blood 1988;72:250A. (Abst 909)

5. Hurst PG, Habib MP, Garewal H, Bluestein M, Paquin P, Greenberg BR. Pulmonary toxicity associated with fludarabine monophosphate Invest New Drugs 1987;5:207-10.

6. Cervantes F, Salgado C, Montserrat E, Rozman C. Fludarabine for prolymphocytic leukaemia and risk of interstitial pneumonitis. Lancet 1990;336:1130. (Lett)

7. Kane GC, McMichael AJ, Patrick H, Erslev AJ. Pulmonary toxicity and acute respiratory failure associated with fludarabine monophosphate. Respir Med 1992;86:261-3.

8. Rosenow EC III. Drug induced pulmonary disease. Disease-a-Month 1994;40:253-310. 


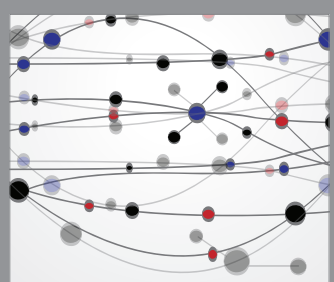

The Scientific World Journal
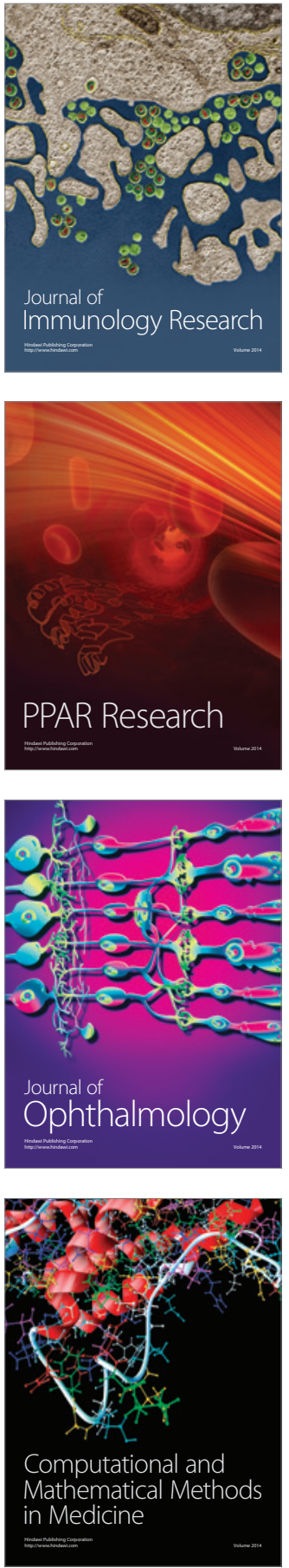

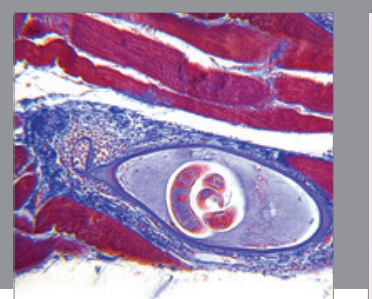

Gastroenterology Research and Practice

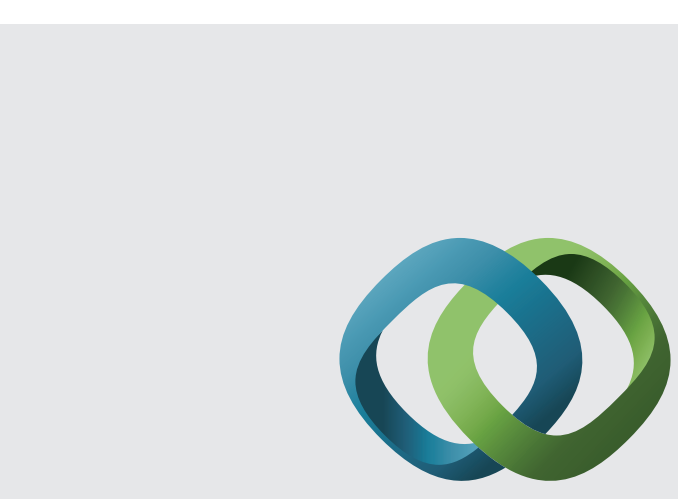

\section{Hindawi}

Submit your manuscripts at

http://www.hindawi.com
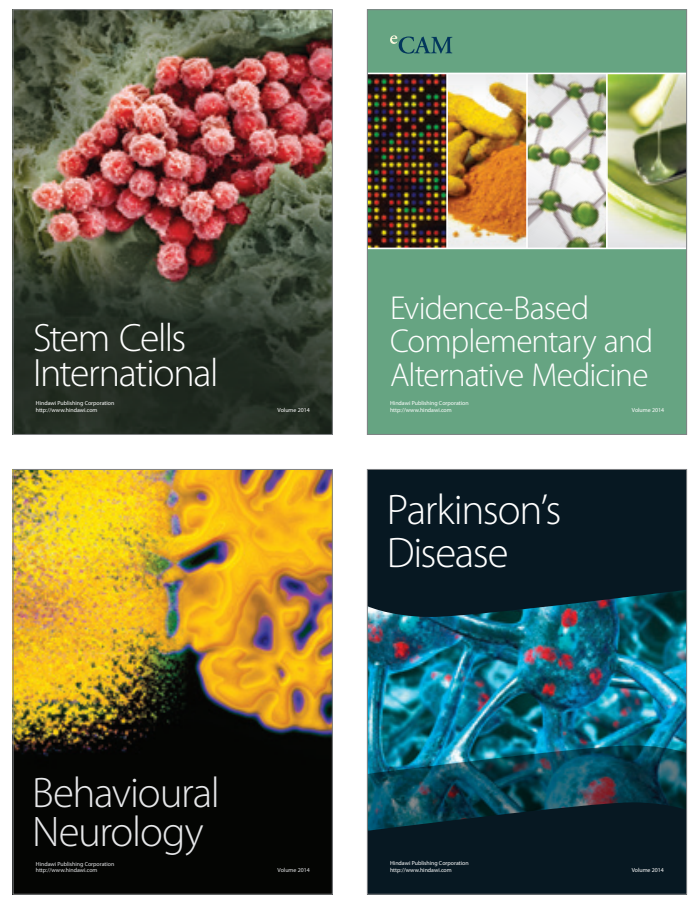
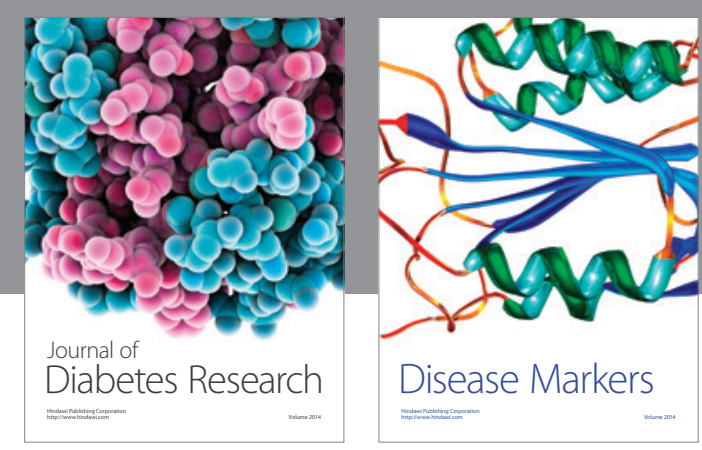

Disease Markers
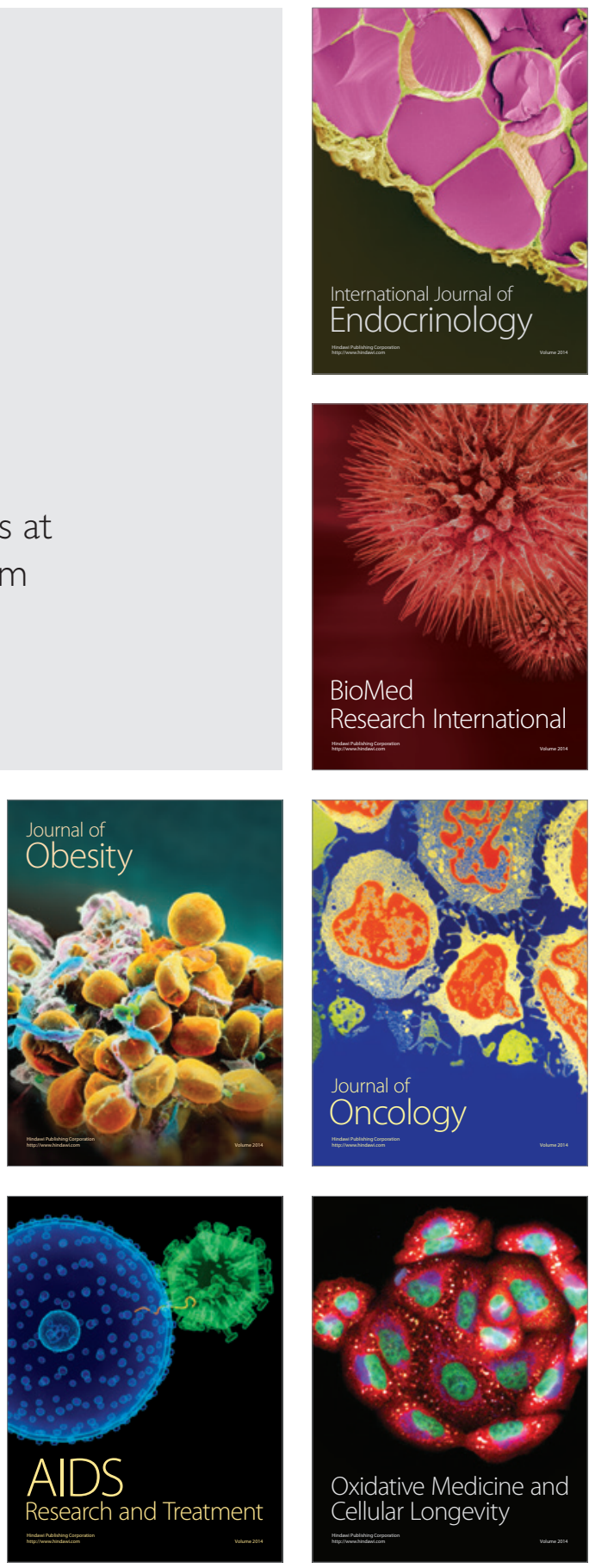